\title{
Adiposity Mediates the Association between the Dietary Inflammatory Index and Markers of Type 2 Diabetes Risk in Middle-Aged Black South African Women
}

\author{
Asanda Mtintsilana ${ }^{1}$, Lisa K. Micklesfield ${ }^{1,2} \oplus$, Elin Chorell ${ }^{3}$, Tommy Olsson ${ }^{3} \oplus$, \\ Nitin Shivappa ${ }^{4,5,6}$, James R Hebert ${ }^{4,5,6}$ (D) Andre P. Kengne ${ }^{7}$ (D) and Julia H. Goedecke ${ }^{1,2,7, *(D)}$ \\ 1 South African Medical Research Council/University of the Witwatersrand Developmental Pathways for \\ Health Research Unit, Department of Paediatrics, Faculty of Health Sciences, University of the \\ Witwatersrand, Johannesburg 2193, South Africa; mtintsilana.asanda@gmail.com (A.M.); \\ Lisa.Micklesfield@wits.ac.za (L.K.M.) \\ 2 Division of Exercise Science and Sports Medicine, Department of Human Biology, University of Cape Town, \\ Cape Town 7725, South Africa \\ 3 Department of Public Health and Clinical Medicine, Umeå University, SE-901 87 Umeå, Sweden; \\ elin.chorell@umu.se (E.C.); Tommy.G.Olsson@umu.se (T.O.) \\ 4 Cancer Prevention and Control Program, University of South Carolina, 915 Greene Street, Suite 200, \\ Columbia, SC 29208, USA; shivappa@mailbox.sc.edu (N.S.); jhebert@sc.edu (J.R.H.) \\ 5 Department of Epidemiology and Biostatistics, University of South Carolina, 915 Greene Street, Suite 400, \\ Columbia, SC 29208, USA \\ 6 Connecting Health Innovations, LLC, 1417 Gregg Street, Columbia, SC 29201, USA \\ 7 Non-Communicable Diseases Research Unit, South African Medical Research Council, Francie Van Zijl, \\ Parow Valley, Cape Town 7505, South Africa; Andre.Kengne@mrc.ac.za \\ * Correspondence: Julia.goedecke@mrc.ac.za; Tel.: +27-21-9380862
}

Received: 20 March 2019; Accepted: 2 May 2019; Published: 31 May 2019

\begin{abstract}
The dietary inflammatory index (DII $\left.{ }^{\circledR}\right)$, a validated tool used to measure the inflammatory potential of the diet, has been associated with metabolic disorders in various settings, but not in African populations. The aim of this study was to investigate whether the DII is associated with markers of type 2 diabetes (T2D) risk, and if this association is mediated by adiposity and/or low-grade inflammation, in black South Africa women. Energy-adjusted-DII (E-DII) scores were calculated in 190 women (median age, 53 years) from the Birth-to-Twenty plus cohort using a validated food frequency questionnaire. Fasting glucose, insulin, $\mathrm{HbA1c}$, and inflammatory cytokines were measured, and an oral glucose tolerance test performed. Basic anthropometry and dual-energy x-ray absorptiometry-derived body fat, including estimate of visceral adipose tissue (VAT) area, were measured. E-DII scores were associated with all markers of T2D risk, namely, fasting glucose and insulin, HbA1c, HOMA2-IR, two-hour glucose and Matsuda index (all $p<0.05)$. After adjusting for age, measures of adiposity, but not inflammatory cytokines, mediated the association between E-DII and markers of T2D risk $(p<0.05)$. Measures of central obesity had proportionally higher (range: 23.5-100\%) mediation effects than total obesity (range: 10-60\%). The E-DII is associated with T2D risk through obesity, in particular central obesity, among black middle-aged South African women.
\end{abstract}

Keywords: DII; diet-induced inflammation; obesity; VAT; mediation; T2D risk; South African women

\section{Introduction}

The rapid increase in the prevalence of non-communicable diseases (NCDs), such as type 2 diabetes (T2D), has become a major health concern, particularly in developing countries, such as South 
Africa (SA) [1-3]. South Africa had a diabetes prevalence of 5.5\% in 2017 [3]. In 2016, diabetes was the second leading cause of mortality in SA after tuberculosis, accounting for $5.5 \%$ of all deaths in both males and females [2]. When classified by sex, diabetes-attributed mortality was worse in females $(7.2 \%)$ than in males $(4.0 \%)$, and ranked first and fifth as the cause of death in females and males in 2016, respectively [2].

The prevalence of obesity and insulin resistance (IR), major risk factors for T2D, is higher in black SA women than in white SA women [4-6]. Of great concern is that urban black SA women are at even greater risk of NCDs compared to their rural counterparts $[7,8]$. Urbanization, which is often accompanied by unhealthy diets and low levels of physical activity, has been identified as the main driver of obesity in developing countries [8-11]. Urban black SA women who follow a typical Western diet characterized by a high intake of fat, sugar and processed meat, and low intake of fruit and vegetables, are more susceptible to NCDs than rural black women who still follow a traditional diet, which is high in fiber, fruit, vegetables and low in animal products $[8,10,11]$.

The relationship between unhealthy diets and NCDs can be attributed to numerous factors, including diet-induced inflammation [12,13]. Indeed, several studies suggest that unhealthy diets may be involved in the pathogenesis of NCDs through their effects on increasing low-grade inflammation [12-14], while healthier diets, such as the Mediterranean diet, which are high in fruits, vegetables and fish, are associated with lower levels of inflammation $[12,13,15]$. Low-grade inflammation is characterized by high circulating levels of pro-inflammatory cytokines, such as c-reactive protein (CRP), tumor necrosis factor- alpha (TNF- $\alpha$ ), interleukins (e.g., IL-6), and infiltration of macrophages in insulin-dependent tissues, which may lead to IR and, subsequently, to T2D [16,17].

The dietary inflammatory index $\left(\mathrm{DII}^{\circledR}\right)$, is a literature-derived dietary tool that measures the inflammatory potential of an individual's diet [14]. Participants with higher DII (i.e., positive values) scores are categorized as having a more pro-inflammatory diet and those with lower DII (i.e., negative values) score represent a more anti-inflammatory diet [14]. The DII has been validated with several inflammatory markers, such as CRP and interleukins [18]; and has recently been associated with markers of glycaemia, namely, fasting glucose, $\mathrm{HbA} 1 \mathrm{c}$, post-load glucose and IR in non-African populations [19-21].

Moreover, participants with higher DII scores were at greater risk of developing prediabetes and T2D compared to participants with lower DII scores [20,21]. Notably, the association between DII and markers of T2D has not been studied in a black African population, whose lifestyle (i.e., diet and socioeconomic status (SES)) differs from white and Hispanic populations. SES (both low and high) and sociocultural factors have thus, through their effects on unhealthy diets and eating behaviors, been associated with NCD risk in black SA communities [22,23]. Furthermore, the black SA population still experiences inadequate micronutrient intake $[10,11]$, in particular micronutrients with putative anti-inflammatory properties, such as vitamin A, niacin, iron and zinc. In contrast, energy intake has not undergone any major change [10,11], suggesting that the quality of the diet, rather than the quantity of food consumed, and changes in energy expenditure may explain the high obesity levels, and, in turn, the susceptibility to T2D risk in urban black SA women.

Use of the DII may contribute to our understanding of the role of diet-induced inflammation in the pathophysiology of T2D, while linking it to markers of low-grade inflammation and obesity. Indeed, the DII has been associated with markers of low-grade inflammation and measures of adiposity (i.e., BMI) and these risk factors have also been suggested to mediate the association between DII and markers of T2D in non-African populations [19].

The aim of this study was to determine whether dietary-induced inflammation, measured by the DII, is associated with markers of T2D risk, and if this association is mediated by adiposity and/or inflammatory markers, in middle-aged and older black SA women. 


\section{Materials and Methods}

\subsection{Study Design}

This cross-sectional study was conducted between 2015 and 2016 and included 190 black female caregivers of the original Birth to Twenty plus (BT20+) cohort, living in Soweto, Johannesburg [24]. The female caregivers were invited to participate if they fulfilled the following inclusion criteria: (1) Female caregiver of the original cohort; (2) provided stored serum samples and blood analyte data (criteria for the larger study); (3) less than 65 years of age; (4) HIV negative on testing; (5) not currently pregnant or lactating. Of those tested $(n=221)$, nine women were excluded from the statistical analyses, due to unreliable energy intake data (i.e., excessive energy intake $(\geq 30,000 \mathrm{~kJ}), n=4)$, lack of food frequency questionnaire (FFQ) data $(n=2)$ and lack of blood samples $(n=3)$. Furthermore, 22 diabetic participants (based on self-report and/or use of T2D medication) were excluded from the analyses, resulting in a final sample size of 190 participants.

The study was approved by the Human Research Ethics Committee (Medical) of the University of the Witwatersrand (M010556 and M150530). The procedures and risks associated with the study were explained to the participants and they all signed the consent form prior to participation in the study. All testing procedures were performed at the South African Medical Research Council (MRC)/University of the Witwatersrand (WITS) Developmental Pathways for Health Research Unit (DPHRU) at the Chris Hani Baragwanath Hospital in Soweto Johannesburg.

\subsection{Body Composition}

Weight and height were measured in subjects wearing only lightweight clothing and without shoes, using a standard scale and stadiometer. Waist (level of umbilicus) and hip (largest gluteal area) circumferences were measured in triplicate and the average was used for statistical analyses.

The International Diabetes Federation waist circumference (WC) threshold (women: $80 \mathrm{~cm}$ ) was used to calculate abdominal obesity (expressed as a percentage) [25]. BMI was calculated as weight $(\mathrm{kg}) /$ height $(\mathrm{m})^{2}$ and classified according to WHO criteria; underweight $\left(<18.5 \mathrm{~kg} / \mathrm{m}^{2}\right)$, normal weight (18.5-24.9 kg/m $\left.{ }^{2}\right)$, overweight $\left(25-29.9 \mathrm{~kg} / \mathrm{m}^{2}\right)$, or obese $\left(\geq 30 \mathrm{~kg} / \mathrm{m}^{2}\right)$ [26]. Whole body composition was measured using dual energy x-ray absorptiometry (DXA; Hologic Discovery-W (S/N 71201), Bedford, MA, USA; software version 13.4.2:7), and included subtotal (whole body minus head) fat mass (expressed in $\mathrm{kg}$ and as a percentage of sub-total fat mass, \%FM). Abdominal visceral adipose tissue (VAT) was estimated using algorithms included in the DXA, and has been shown to correlate with clinical computed tomography [27]. Body composition of participants exceeding the scan field limits was calculated using the arm-replacement [28]. The coefficient of variations were $1 \%$ and $<2 \%$ for fat-free soft tissue mass and total fat mass, respectively.

\subsection{Socio-Demographic Assessment}

A demographic questionnaire, written in English, was administered in the participant's language of choice (i.e., English, IsiXhosa, IsiZulu, SeSotho, Sepedi, SeTswana, TshiVenda, XiTsonga, SiSwati) and included measures of SES, including housing density, asset index, household food insecurity, marital status, educational assessment, and employment. Housing density was defined as the number of persons per room living in the household. Asset index was based on 12 items (e.g., motor vehicle, washing machine, TV) reflecting individual and household wealth. Food insecurity was assessed using a validated nine question Household Food Insecurity Access Scale [29]. The responses to the nine-questionnaire scale are summed to create a food security score, with a maximum score of 27. The higher the score the more food insecurity the household experienced, whereas the lower the score the less food insecurity experienced in the past four weeks [29]. Education was categorized by grades passed. Participants were categorized as being married or not, employed or unemployed. Moreover, questions on lifestyle factors, including smoking and alcohol intake were recorded. Physical activity and sedentary behavior were measured using ActiGraph GT3X-Plus triaxial accelerometers (ActiGraph 
GTX3+, ActiGraph LLC, Pensacola, Florida) and activPAL devices (activPAL3c, PAL Technologies Ltd., Glasgow, UK), respectively, as described previously [30].

\subsection{The Dietary Inflammatory Index (DII $\left.{ }^{\circledR}\right)$}

The DII scores for all the participants in the current study were calculated from the dietary intake data collected using a seven-day food frequency questionnaire [31,32]. The development and validation of the DII have been described in detail elsewhere $[14,18]$. The DII is a literature-derived, validated tool used to measure the inflammatory potential of the diet based on extensive literature search performed to identify peer-reviewed primary research articles published through 2010 that reported the association between various dietary factors and six inflammatory markers (IL-1 $\beta$, IL-4, IL-6, IL-10, TNF $\alpha$, and CRP). A total of 1943 qualifying articles were reviewed, indexed, and scored to derive the component-specific inflammatory effect score for 45 dietary factors (i.e., components of DII), comprising macronutrients and micronutrients, as well as some bioactive components [14]. These FFQ-derived food and nutrient intake data were first adjusted for total energy (i.e., per 1000 kilocalories) and standardized by creating a $\mathrm{z}$ score for each component using mean and standard deviation data from a global energy-adjusted dietary database comprised of dietary intake from 11 populations living in different regions of the world. To reduce the effect of right-skewing the $\mathrm{z}$ scores for each DII component were converted to proportions (i.e., values from 0 to 1 ) and then each proportion was centered by doubling and subtracting 1 . From this, the energy-adjusted standardized dietary intake (expressed as centered proportions) was then multiplied by the corresponding literature-derived inflammatory effect score for each DII component. Individual scores from each DII component were then summed to determine the overall E-DII score for each individual [14]. A higher (i.e., more positive) score indicates a more pro-inflammatory diet and a lower score (i.e., more negative) represents a more anti-inflammatory diet. The DII scores can range between 7.98 (maximally pro-inflammatory) and -8.87 (maximally anti-inflammatory) [14]. Steps involved in the DII calculations are described in Figure 1. 
Review of articles published from 1950 to 2010 resulting in 1943 studies linking a total of 45 food parameters with inflammatory biomarkers.

A score for each food parameter was calculated giving:

$\mathbf{+ 1}$ to each article if the effects were pro-inflammatory (significantly increased IL-1 $\beta$, IL-6, TNF- $\alpha$ or CRP, or decreased IL-4 or IL-10),

$\mathbf{- 1}$ if the effects were anti-inflammatory (significantly decreased IL-1 $\beta$, IL-6, TNF- $\alpha$ or CRP, or increased IL-4 or IL-10),

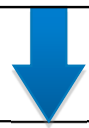

The score for each food parameter was weighted according to the study design. The weights were 10 (experimental design), 8 (observational), 7 (case-control), 6 (cross-sectional), 5 (experimental with animals), 3 (cell culture).

\section{(20)

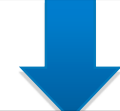 \\ A food parameter-specific overall inflammatory effect score was calculated by subtracting the anti-inflammatory fraction from the pro-inflammatory fraction. This score was corrected if the total weighted number of articles was $<236$. In these cases the raw overall inflammatory score is multiplied by the total weighted number of articles divided by 236 .}

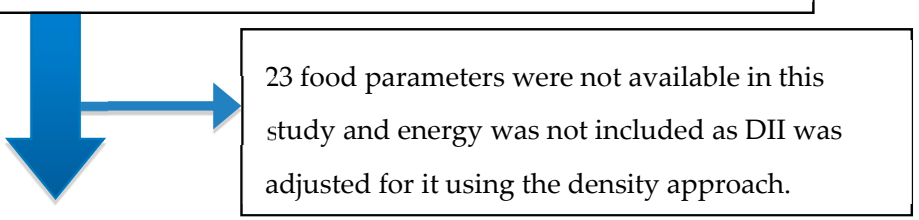

Z-score and centred-percentiles for each of the 21 food parameters for each participant of this study were calculated based on the average and standard deviation for each food parameter obtained from the global database which was created from the consumption of the original 45 food parameters from 11 countries from around the world.

The centred percentile for each food parameter was multiplied by the respective "overall food parameter-specific inflammatory effect score" to obtain the "food parameter-specific DII score".

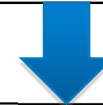

All of the "food parameter-specific DII scores" are summed to create the "overall DII score" for each individual.

Figure 1. Sequence of steps in creating the energy-adjusted- DII (dietary inflammatory index) in middle-aged black South African women.

For the current study, a total of 21 out of 45 food parameters were available in our dietary intake database, and were thus used for the analysis of the DII score. The 21 food parameters included protein, 
fat, SFA, monounsaturated fatty acid (MUFA), polyunsaturated fatty acid (PUFA), carbohydrate, cholesterol, alcohol, fiber, iron, magnesium, Zinc, vitamin A (retinol equivalent), thiamine, riboflavin, niacin, vitamin B6, vitamin B12, vitamin C, vitamin D and vitamin E. Participants with E-DII scores greater than 0 were classified as the positive E-DII group (reflecting pro-inflammatory diet; $n=83$ ) and those with E-DII scores less than 0 were classified as negative E-DII group (indicating anti-inflammatory diet; $n=107)$. There were no participants with E-DII score equal to 0 .

\subsection{Biochemical Analysis}

Blood samples were drawn in the fasted state (12 hours, overnight) for the subsequent determination of glycated hemoglobin $(\mathrm{HbA} 1 \mathrm{c})$, plasma glucose and serum insulin concentrations. Thereafter, all participants completed a standard oral glucose tolerance test (OGTT). In brief, participants ingested $75 \mathrm{~g}$ of anhydrous glucose dissolved in $250 \mathrm{~mL}$ water, within 5 minutes. Following the glucose ingestion, blood samples were taken at 30,60,90 and $120 \mathrm{~min}$. The samples were centrifuged at $3000 \mathrm{rpm}$ for $10 \mathrm{~min}$ at $4{ }^{\circ} \mathrm{C}$, the plasma was stored at $-20^{\circ} \mathrm{C}$ for subsequent analysis of glucose concentrations, and the serum was stored at $-80^{\circ} \mathrm{C}$ for the analysis of insulin concentrations and inflammatory markers. Plasma glucose concentrations were analyzed on the Randox RX Daytona Chemistry Analyzer using enzymatic methods (Randox Laboratories Ltd., London, UK). Serum insulin assays were analyzed on the Immulite ${ }^{\circledR} 1000$ Immunoassay System (Siemens Chemiluminescent Healthcare GmbH, Henkestr, Germany). HbA1c levels were measured on whole blood samples using the D-10 ${ }^{\mathrm{TM}}$ Hemoglobin Analyzer (Bio-Rad Laboratories, Inc. Hercules, CA, USA). Inflammatory cytokines, namely, IL-6, IL-1ra, IL-8, IL-10, IL15, monocyte chemotactic protein (MCP)1, interferon (IFN) gamma, and TNF- $\alpha$ were measured using Milliplex MAP MAG Human Cytokine kit (Merck, Johannesburg, South Africa) and xMAP technology (Luminex, Austin, Texas) according to the manufacturer's instructions. Out of the eight cytokines measured, only TNF- $\alpha, 1 \mathrm{~L}-8$ and MCP-1 were detectable in all the samples, while the rest were below the detectable range, and therefore not included in the analyses.

Insulin resistance (IR) was calculated from fasting glucose and insulin using the Homeostasis Model Assessment (HOMA2-IR) calculator v2.2.3 [33]. Fasting and OGTT glucose and insulin data $(0,30,60,90$ and 120) were used to calculate insulin sensitivity using the Matsuda Index web calculator [34]. Participants with missing glucose and insulin data from the OGTT $(n=17)$ were excluded from this parameter.

\subsection{Statistical Analysis}

The Shapiro-Wilk test was used to assess the distribution of continuous variables. Normally distributed data are presented as mean \pm standard deviation (SD), skewed variables are represented as medians and 25th-75th percentiles, and categorical data are presented as frequency (\%). Moreover, Levene's robust test was used to test for equal variance between groups. Differences in measures of SES, lifestyle/behavioral factors, body composition and markers of T2D risk between the positive and negative E-DII groups were examined using the Student's $t$ test and the Wilcoxon rank-sum (Mann-Whitney), or the Chi square test or Fisher exact test as appropriate. To determine whether the E-DII was associated with markers of T2D risk (fasting glucose and insulin, HbA1c, HOMA2-IR, two-hour glucose and OGTT-derived insulin sensitivity), or whether the association was mediated by adiposity (WC, BMI, VAT and body fat mass) and/or inflammatory markers (TNF- $\alpha$, IL-8 and MCP-1), a simple mediation analysis was completed (Figure 2a,b) [35]. In each model, an independent, a dependent and a mediator variable were included in order to calculate the unstandardized regression coefficients and generate three outputs, namely, total, direct and indirect effects [36]. Path c, represents the simple total effect of E-DII on markers of T2D without adjusting for mediators (Figure 2a). Path $\alpha$ represents the regression coefficient between the independent variable (i.e., E-DII) and the mediator variable (e.g., VAT) (Figure 2a). The regression coefficient of Path $\beta$ represents the effect of the mediator variable on the markers of T2D (Figure 2b). The product of regression coefficients $\alpha$ and $\beta(\alpha \beta)$ represents the mediated effect (indirect effect) of E-DII on markers of T2D through the mediator variable 
(Figure 2b). Path $c^{\prime}$ represents the direct effect of E-DII on markers of T2D after controlling for the effect of the mediator (Figure 2b).

(a)

\section{Path c}

Total effects of E-DII without mediators

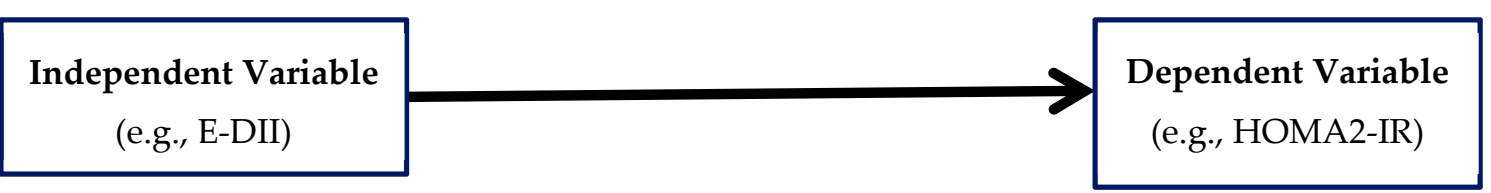

(b)

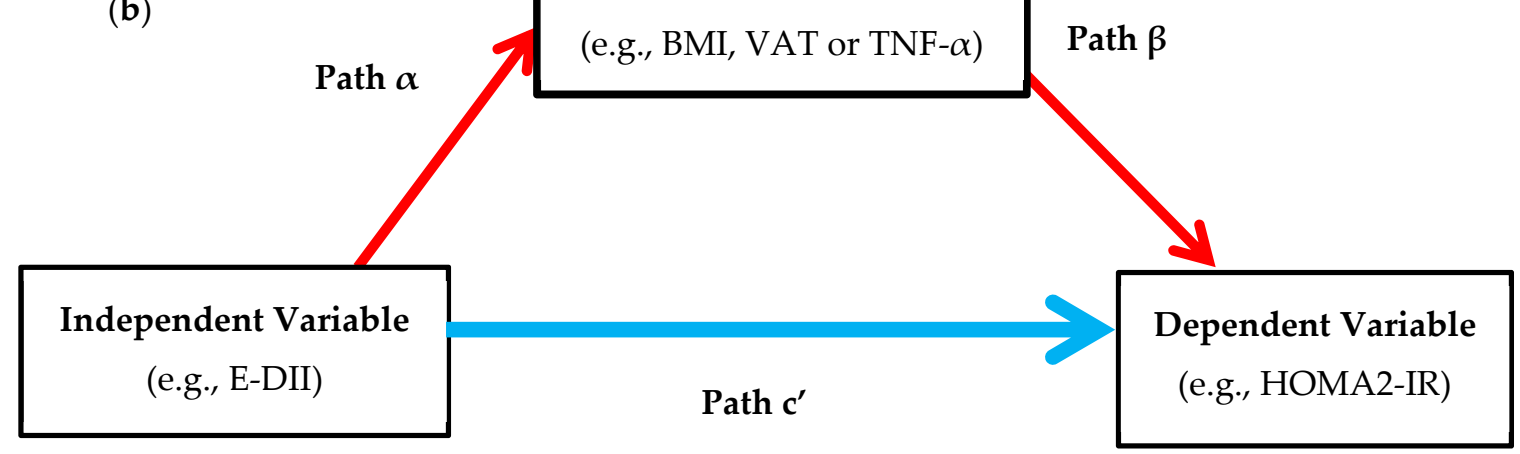

Figure 2. Represent a single mediator model used to test the association between the energy-adjusted-dietary inflammatory index (E-DII) (i.e., independent variable) and markers of type 2 diabetes (T2D) (i.e., dependent variable), body composition and inflammatory cytokines as mediators: (a) Path c, represents the simple total effect of E-DII on markers of T2D, without adjusting for mediators; (b) Represents the direct (Path $\mathbf{c}^{\prime}$ ) and indirect effects (product of Paths $\alpha$ and $\beta, \alpha \beta$ ) of E-DII on markers of T2D after controlling for the effect of the mediator. BMI, body mass index; E-DII, energy-adjusted-dietary inflammatory index; HOMA2-IR, Homeostasis Model Assessment- insulin resistance; TNF- $\alpha$, tumor necrosis factor alpha; VAT, visceral adipose tissue.

Bootstrapping (5000 replications) was used to generate normal-based bootstrapped confidence intervals around the indirect effect. Full or complete mediation is present when the total effect and indirect effects are significant, while the direct effect (path $c^{\prime}$ ) is non-significant. When the total and indirect effects are significant, and the direct effect remains significant, partial or incomplete mediation has occurred. Inconsistent mediation is present when neither total nor direct effect is significant and $\alpha \beta$ is significant. The proportion of the mediation effect was calculated using the following equation: $\left[\alpha \beta /\left(\alpha \beta+c^{\prime}\right)\right]$. The Matsuda Index was modelled as an inverse variable in order to calculate the proportion of mediation effect; thus, the regression coefficients are positive instead of negative. All single mediation analysis models were adjusted for age. All data were analyzed using Stata ${ }^{\circledR}$ (Version 13.1, Statcorp, College Station, TX, USA). A test was considered statistically significant if $p \leq 0.05$.

\section{Results}

\subsection{Participants' Characteristics According to E-DII Score}

The median (25th-75th percentiles) age of the total sample was 53 (48-59) years. The E-DII ranged from +1.92 (most pro-inflammatory) to -2.51 (most anti-inflammatory). Participants were grouped based on whether they had a positive $(n=83 ; 43.7 \%)$ or negative $(n=107 ; 56.3 \%)$ E-DII score (Table 1$)$. 
Table 1. Body composition and clinical characteristics of the participants according to E-DII scores.

\begin{tabular}{|c|c|c|c|c|}
\hline & Overall & Negative E-DII Group $(n=107)$ & Positive E-DII Group ( $n=83$ ) & $p$ Value \\
\hline E-DII score (minimum-maximum value) & $-2.51-1.92$ & $-2.51-0.01$ & $0.01-1.92$ & \\
\hline E-DII & $-0.2 \pm 1.0$ & $-0.9 \pm 0.6$ & $0.6 \pm 0.5$ & \\
\hline Age (years) & $53(48-59)$ & $54(49-59)$ & $54(49-60)$ & 0.87 \\
\hline \multicolumn{5}{|l|}{ Anthropometry } \\
\hline Height $(\mathrm{m})$ & $1.6 \pm 0.1$ & $1.6 \pm 0.1$ & $1.6 \pm 0.1$ & 0.93 \\
\hline Weight (kg) & $84.7(73.6-96.8)$ & $84.0(70.4-94.2)$ & $87.2(76.4-100.2)$ & 0.15 \\
\hline BMI $\left(\mathrm{kg} / \mathrm{m}^{2}\right)$ & $33.5(29.8-38.8)$ & $33.5(29.0-37.2)$ & $34.2(30.2-40.1)$ & 0.23 \\
\hline \multicolumn{5}{|l|}{ BMI categories, $n(\%)$} \\
\hline Normal weight & $18(9.5)$ & $10(9.4)$ & $8(9.6)$ & \multirow{3}{*}{0.46} \\
\hline Overweight & $30(15.8)$ & $20(18.7)$ & $10(12.1)$ & \\
\hline Obese & $142(74.7)$ & $77(72.0)$ & $65(78.3)$ & \\
\hline Waist circumference $(\mathrm{cm})$ & $100.1 \pm 13.0$ & $98.3 \pm 12.3$ & $102.4 \pm 13.7$ & 0.03 \\
\hline \multicolumn{5}{|l|}{ Waist circumference-abdominal obesity, $n(\%)$} \\
\hline Waist circumference $(\geq 80 \mathrm{~cm})$ & $177(93.2)$ & $100(93.5)$ & $77(92.8)$ & \multirow{2}{*}{0.85} \\
\hline Waist circumference $(<80 \mathrm{~cm})$ & $13(6.8)$ & $7(6.5)$ & $6(7.2)$ & \\
\hline Hip circumference $(\mathrm{cm})$ & $121.8 \pm 14.5$ & $121.0 \pm 14.3$ & $122.9 \pm 14.7$ & 0.21 \\
\hline WHR & $0.8 \pm 0.1$ & $0.8 \pm 0.1$ & $0.8 \pm 0.1$ & 0.04 \\
\hline \multicolumn{5}{|l|}{ Body composition and body fat distribution } \\
\hline Body Fat mass $(\mathrm{kg})$ & $39.6(31.5-48.2)$ & $38.7(29.8-46.6)$ & $40.9(33.1-50.8)$ & 0.12 \\
\hline Body fat $(\%)$ & $50.7(46.2-54.0)$ & $50.1(44.8-53.8)$ & $51.5(47.0-54.6)$ & 0.09 \\
\hline VAT area $\left(\mathrm{cm}^{2}\right)$ & $165 \pm 62$ & $155 \pm 59$ & $178 \pm 64$ & 0.01 \\
\hline SAT area $\left(\mathrm{cm}^{2}\right)$ & $507 \pm 157$ & $495 \pm 154$ & $521 \pm 161$ & 0.27 \\
\hline \multicolumn{5}{|l|}{ Clinical biomarkers } \\
\hline Fasting glucose (mmol/L) & $4.9(4.3-5.5)$ & $4.8(4.3-5.4)$ & $5.1(4.3-5.7)$ & 0.07 \\
\hline Fasting insulin $(\mathrm{mUI} / \mathrm{mL})$ & $10.4(5.7-18.0)$ & $9.8(5.7-15.7)$ & $11.7(5.9-18.9)$ & 0.27 \\
\hline Two-hour glucose (mmol/L) & $6.7(5.3-8.1)$ & $6.3(5.3-7.8)$ & $7.2(6.0-8.6)$ & 0.02 \\
\hline $\mathrm{HbA1c}(\%)$ & $5.5(5.1-5.7)$ & $5.4(5.1-5.7)$ & $5.5(5.2-5.8)$ & 0.48 \\
\hline HOMA2-IR & $1.5(0.8-2.6)$ & $1.4(0.8-2.2)$ & $1.7(0.8-2.7)$ & 0.23 \\
\hline Matsuda Index (OGTT-derived insulin sensitivity) & $3.6(2.1-6.0)$ & $3.9(2.4-6.1)$ & $2.9(1.9-5.6)$ & 0.10 \\
\hline TNF- $\alpha(\mathrm{pg} / \mathrm{mL})$ & $11.0(8.0-15.1)$ & $9.9(7.8-14.2)$ & $12.1(8.7-16.7)$ & 0.03 \\
\hline $\mathrm{IL}-8(\mathrm{pg} / \mathrm{mL})$ & $5.4(2.4-11.4)$ & $4.8(2.6-8.9)$ & $6.3(2.2-12.6)$ & 0.16 \\
\hline MCP-1 (pg/mL) & $436.2(325.2-627.5)$ & $449.9(321.2-664.7)$ & $422.6(330.1-595.7)$ & 0.71 \\
\hline
\end{tabular}

Data presented as means \pm SD or median (25th-75th percentiles). BMI, body mass index; DII, dietary inflammatory index; HbA1c, glycated hemoglobin; HOMA2-IR, Homeostasis Model Assessment- insulin resistance; IL-8; Interleukin-8, MCP-1, monocyte chemotactic protein 1; OGTT, oral glucose tolerance test. SAT, subcutaneous adipose tissue; TNF- $\alpha$, tumor necrosis factor alpha; VAT, visceral adipose tissue; WC, waist circumference; WHR, waist-to-hip ratio. 
While BMI and total body fat did not differ between groups, waist circumference, WHR and VAT were higher in the positive E-DII group compared to the negative E-DII group. Furthermore, participants with a positive E-DII score had higher two-hour glucose and TNF- $\alpha$ levels than those with a negative E-DII score $(p<0.05)$. There were no significant differences in any of the fasting or OGTT-derived measures of T2D risk, and inflammatory markers (IL-8 and MCP-1) did not differ between the groups (Table 1).

Comparisons of measures of SES and lifestyle/behavioral factors, and nutrient intakes between the two E-DII score groups are presented in Tables S1 and S2, respectively. There were no differences in any of the measures of SES and lifestyle/behavioral factors, including physical activity and sedentary behaviors between the two E-DII score groups (Table S1). A median score of 9 out of 12 possible household items (e.g., motor vehicle and washing machine) was reported for the participants. Furthermore, the participants scored an average of 4 (range: 0-9) on the Household Food Insecurity Access Scale, indicating that the participant/household experienced less food insecurity for the measured period.

Energy and carbohydrate intake (except when carbohydrate is expressed as a percentage of energy intake), alcohol and cholesterol levels did not differ between groups (Table S2). In contrast, protein was lower, but total fat, SFA, MUFA and PUFA (both in grams and percentage of energy intake) were higher in the positive E-DII score group than the negative E-DII score group (all $p<0.05$ ) (Table S2).

Vitamins A, B12 and D, and riboflavin did not differ between the groups, whereas fiber intake and the rest of the minerals (e.g., iron, magnesium and zinc) and vitamins (e.g., vitamin B6, C and E) were lower in the positive DII score compared to the negative DII score group $(p<0.05)$ (Table S2).

\subsection{Associations between E-DII and Markers of T2D Risk in Black SA Women}

The unstandardized regression coefficients of the total effect of E-DII on markers of T2D risk without (Path c) and with body composition and inflammatory cytokines as mediators (Path c') are presented in Tables 2 and 3, respectively. All the models were adjusted for the potential effects of age. E-DII was positively associated with all markers of T2D risk, namely, fasting glucose and insulin, $\mathrm{HbA1c}$, HOMA2-IR, two-hour glucose levels and Matsuda Index (i.e., OGTT-derived insulin sensitivity) (all $p<0.05$ ). 
Table 2. Direct and indirect effects of E-DII on markers of T2D risk with body composition as mediators in black South African (SA) women.

\begin{tabular}{|c|c|c|c|c|c|c|c|}
\hline \multirow{2}{*}{$\begin{array}{c}\text { Variables } \\
\text { Mediators and Outcomes }\end{array}$} & \multicolumn{2}{|c|}{ Total Effect (c) } & \multicolumn{2}{|c|}{ Direct Effect (c') } & \multicolumn{2}{|c|}{ Indirect Effect $(\alpha \beta)$} & \multirow{2}{*}{$\begin{array}{c}\begin{array}{c}\text { Proportion of } \\
\text { Mediation }\end{array} \\
\% \\
\end{array}$} \\
\hline & Estimate $(95 \% \mathrm{CI})$ & $p$-Value & Estimate $(95 \% \mathrm{CI})$ & $p$-value & $95 \% \mathrm{CI}$ & $p$-value & \\
\hline Fasting glucose (mmol/L) & $0.17(0.01-0.34)$ & 0.04 & & & & & \\
\hline via waist circumference $(\mathrm{cm})$ & & & $0.12(-0.07-0.32)$ & 0.21 & $0.04(-0.03-0.12)$ & 0.25 & 23.5 \\
\hline via BMI $\left(\mathrm{kg} / \mathrm{m}^{2}\right)$ & & & $0.13(-0.05-0.32)$ & 0.16 & $0.04(-0.03-0.10)$ & 0.28 & 23.5 \\
\hline via VAT $\left(\mathrm{cm}^{2}\right)$ & & & $0.07(-0.10-0.24)$ & 0.42 & $0.10(0.00-0.20)$ & 0.04 & 58.8 \\
\hline via body fat mass $(\mathrm{kg})$ & & & $0.14(-0.05-0.33)$ & 0.14 & $0.03(-0.03-0.08)$ & 0.371 & 17.6 \\
\hline Fasting insulin $(\mathrm{mUI} / \mathrm{mL})$ & $1.70(0.45-2.96)$ & 0.008 & & & & & \\
\hline via waist circumference $(\mathrm{cm})$ & & & $0.38(-0.87-1.63)$ & 0.55 & $1.32(0.40-2.25)$ & 0.005 & 77.6 \\
\hline via $B M I\left(k g / \mathrm{m}^{2}\right)$ & & & $0.71(-0.51-1.93)$ & 0.26 & $0.99(0.20-1.78)$ & 0.01 & 58.2 \\
\hline via VAT $\left(\mathrm{cm}^{2}\right)$ & & & $0.08(-1.19-1.35)$ & 0.90 & $1.62(0.60-2.64)$ & 0.002 & 95.3 \\
\hline via body fat mass $(\mathrm{kg})$ & & & $0.89(-0.30-2.09)$ & 0.14 & $0.81(0.11-1.51)$ & 0.02 & 47.6 \\
\hline HbA1c $(\%)$ & $0.10(0.01-0.18)$ & 0.02 & & & & & \\
\hline via waist circumference $(\mathrm{cm})$ & & & $0.07(-0.02-0.15)$ & 0.14 & $0.03(-0.00-0.06)$ & 0.07 & 30.0 \\
\hline via $B M I\left(\mathrm{~kg} / \mathrm{m}^{2}\right)$ & & & $0.08(-0.01-0.16)$ & 0.08 & $0.02(-0.01-0.05)$ & 0.13 & 20.0 \\
\hline via VAT $\left(\mathrm{cm}^{2}\right)$ & & & $0.05(-0.02-0.14)$ & 0.18 & $0.04(0.00-0.08)$ & 0.04 & 40.0 \\
\hline via body fat mass $(\mathrm{kg})$ & & & $0.08(-0.00-0.17)$ & 0.06 & $0.01(-0.01-0.04)$ & 0.19 & 10.0 \\
\hline HOMA2-IR & $0.25(0.07-0.44)$ & 0.008 & & & & & \\
\hline via waist circumference $(\mathrm{cm})$ & & & $0.05(-0.15-0.26)$ & 0.59 & $0.20(0.05-0.35)$ & 0.009 & 80.0 \\
\hline via $B M I\left(\mathrm{~kg} / \mathrm{m}^{2}\right)$ & & & $0.10(-0.09-0.30)$ & 0.30 & $0.15(0.02-0.28)$ & 0.02 & 60.0 \\
\hline via VAT $\left(\mathrm{cm}^{2}\right)$ & & & $0.00(-0.20-0.21)$ & 0.97 & $0.25(0.08-0.41)$ & 0.003 & 100.0 \\
\hline via body fat mass $(\mathrm{kg})$ & & & $0.13(-0.05-0.32)$ & 0.16 & $0.12(0.01-0.23)$ & 0.03 & 48.0 \\
\hline Two-hour glucose $(\mathrm{mmol} / \mathrm{L})$ & $0.48(0.10-0.86)$ & 0.01 & & & & & \\
\hline via waist circumference $(\mathrm{cm})$ & & & $0.34(-0.08-0.76)$ & 0.11 & $0.14(-0.01-0.028)$ & 0.06 & 29.2 \\
\hline via BMI $\left(\mathrm{kg} / \mathrm{m}^{2}\right)$ & & & $0.39(-0.02-0.81)$ & 0.06 & $0.09(-0.04-0.21)$ & 0.17 & 18.8 \\
\hline via VAT $\left(\mathrm{cm}^{2}\right)$ & & & $0.21(-0.15-0.56)$ & 0.26 & $0.27(0.08-0.47)$ & 0.006 & 56.3 \\
\hline via body fat mass $(\mathrm{kg})$ & & & $0.42(0.00-0.83)$ & 0.05 & $0.06(-0.04-0.17)$ & 0.27 & 12.5 \\
\hline Matsuda Index * & $0.05(0.02-0.09)$ & 0.003 & & & & & \\
\hline via waist circumference $(\mathrm{cm})$ & & & $0.01(-0.04-0.06)$ & 0.62 & $0.04(0.00-0.08)$ & 0.03 & 80.0 \\
\hline via $B M I\left(\mathrm{~kg} / \mathrm{m}^{2}\right)$ & & & $0.02(-0.02-0.07)$ & 0.31 & $0.03(-0.00-0.06)$ & 0.05 & 60.0 \\
\hline via VAT $\left(\mathrm{cm}^{2}\right)$ & & & $-0.01(-0.06-0.05)$ & 0.85 & $0.05(0.01-0.10)$ & 0.01 & 100.0 \\
\hline via body fat mass $(\mathrm{kg})$ & & & $0.03(-0.01-0.07)$ & 0.16 & $0.02(-0.00-0.05)$ & 0.08 & 40.0 \\
\hline
\end{tabular}

Regression coefficients $\mathrm{c}, \alpha, \beta$ and $\mathrm{c}^{\prime}$ are shown in Figure 1a,b. All estimates were adjusted for the potential effects of age. BMI, body mass index; DII, dietary inflammatory index; HbA1c, glycated hemoglobin; HOMA2-IR, Homeostasis Model Assessment- insulin resistance; VAT, visceral adipose tissue. * Matsuda index was modelled as an inverse variable in order to calculate the proportion of mediation effect, thus the regression coefficients are positive instead of negative. 
Table 3. Total, direct and indirect effects of E-DII on markers of T2D risk with inflammatory cytokines as mediators in black SA women.

\begin{tabular}{|c|c|c|c|c|c|c|}
\hline \multirow{2}{*}{$\begin{array}{r}\text { Variables } \\
\text { Mediators and Outcomes }\end{array}$} & \multicolumn{2}{|c|}{ Total Effect (c) } & \multicolumn{2}{|c|}{ Direct Effect (c') } & \multicolumn{2}{|c|}{ Indirect Effect $(\alpha \beta)$} \\
\hline & Estimate $(95 \% \mathrm{CI})$ & $p$-Value & Estimate $(95 \% \mathrm{CI})$ & $p$-Value & Estimate $(95 \% \mathrm{CI})$ & $p$-Value \\
\hline Fasting glucose $(\mathrm{mmol} / \mathrm{L})$ & $0.17(0.01-0.34)$ & 0.04 & & & & \\
\hline via TNF- $\alpha(\mathrm{pg} / \mathrm{mL})$ & & & $0.16(-0.01-0.33)$ & 0.07 & $0.01(-0.01-0.04)$ & 0.28 \\
\hline via IL-8 $(\mathrm{pg} / \mathrm{mL})$ & & & $0.17(0.01-0.34)$ & 0.04 & $-0.00(-0.01-0.01)$ & 0.71 \\
\hline via MCP-1 (pg/mL) & & & $0.16(0.01-0.32)$ & 0.04 & $0.01(-0.01-0.03)$ & 0.43 \\
\hline Fasting insulin (mUI/mL) & $1.70(0.45-2.96)$ & 0.008 & & & & \\
\hline via TNF- $\alpha(\mathrm{pg} / \mathrm{mL})$ & & & $1.55(0.32-2.78)$ & 0.01 & $0.15(-0.06-0.36)$ & 0.17 \\
\hline via IL-8 (pg/mL) & & & $1.72(0.47-2.97)$ & 0.007 & $-0.02(-0.14-0.11)$ & 0.77 \\
\hline via MCP-1 (pg/mL) & & & $1.66(0.43-2.88)$ & 0.008 & $0.04(-0.13-0.21)$ & 0.63 \\
\hline HbA1c (\%) & $0.10(0.01-0.18)$ & 0.02 & & & & \\
\hline via TNF- $\alpha(\mathrm{pg} / \mathrm{mL})$ & & & $0.11(0.02-0.19)$ & 0.01 & $-0.01(-0.02-0.00)$ & 0.15 \\
\hline via IL-8 $(\mathrm{pg} / \mathrm{mL})$ & & & $0.10(0.02-0.18)$ & 0.02 & $-0.00(-0.01-0.00)$ & 0.49 \\
\hline via MCP-1 (pg/mL) & & & $0.10(0.02-0.18)$ & 0.02 & $-0.00(-0.01-0.01)$ & 0.92 \\
\hline HOMA2-IR & $0.25(0.07-0.44)$ & 0.008 & & & & \\
\hline via TNF- $\alpha(\mathrm{pg} / \mathrm{mL})$ & & & $0.23(0.05-0.41)$ & 0.02 & $0.02(-0.01-0.06)$ & 0.15 \\
\hline via IL-8 (pg/mL) & & & $0.26(0.07-0.44)$ & 0.007 & $-0.00(-0.02-0.02)$ & 0.75 \\
\hline via MCP-1 (pg/mL) & & & $0.25(0.07-0.43)$ & 0.007 & $0.01(-0.02-0.03)$ & 0.59 \\
\hline Two-hour glucose (mmol/L) & $0.48(0.10-0.86)$ & 0.01 & & & & \\
\hline via TNF- $\alpha(\mathrm{pg} / \mathrm{mL})$ & & & $0.46(0.07-0.85)$ & 0.02 & $0.02(-0.02-0.07)$ & 0.31 \\
\hline via IL-8 (pg/mL) & & & $0.49(0.11-0.87)$ & 0.01 & $-0.01(-0.04-0.01)$ & 0.42 \\
\hline via $\mathrm{MCP}-1(\mathrm{pg} / \mathrm{mL})$ & & & $0.46(0.08-0.83)$ & 0.02 & $0.03(-0.04-0.09)$ & 0.41 \\
\hline Matsuda Index * & $0.05(0.02-0.09)$ & 0.003 & & & & \\
\hline via TNF- $\alpha(\mathrm{pg} / \mathrm{mL})$ & & & $0.05(0.01-0.08)$ & 0.007 & $0.00(-0.00-0.01)$ & 0.23 \\
\hline via IL-8 (pg/mL) & & & $0.05(0.02-0.09)$ & 0.003 & $-0.00(-0.00-0.00)$ & 0.61 \\
\hline via MCP-1 (pg/mL) & & & $0.05(0.02-0.09)$ & 0.003 & $0.00(-0.00-0.01)$ & 0.67 \\
\hline
\end{tabular}

Regression coefficients $\alpha, \beta$ and $c^{\prime}$ are shown in Figure 2a,b. All estimates were adjusted for the potential effects of age. DII, dietary inflammatory index; HbA1c, glycated hemoglobin; HOMA2-IR, Homeostasis Model Assessment- insulin resistance; IL-8; Interleukin-8, MCP-1, monocyte chemotactic protein 1; TNF- $\alpha$, tumor necrosis factor alpha. * Matsuda index was modelled as an inverse variable in order to calculate the proportion of mediation effect, thus the regression coefficients are positive instead of negative. 
The association between the proposed mediators and E-DII (path $\alpha$ ) are presented in Table 4 , while the associations between the potential mediators and the outcome, markers of T2D risk (path $\beta$ ), are presented in Table 5. Path $\alpha$ showed that E-DII had a significant association with all body composition measures, whereas only TNF- $\alpha$ associated with E-DII among the pro-inflammatory cytokines (Table 4). Analysis of Path $\beta$, showed that all the body composition measures were significantly associated with fasting insulin, HOMA2-IR and Matsuda Index Table 5). Furthermore, both WC and VAT (measures of central obesity) were positively associated with $\mathrm{HbA1c}$ and two-hour glucose load, with only VAT significantly associated with fasting glucose. In contrast, none of the inflammatory cytokines were significantly associated with markers of T2D risk (Table 5).

Table 4. Regression coefficients (95\% CIs) for the association between E-DII and measures of body composition and inflammatory cytokines in black SA women.

\begin{tabular}{lccc}
\hline \multicolumn{1}{c}{ Mediator Variables } & Estimate & $\mathbf{9 5 \% C I}$ & $p$-Value \\
\hline Waist circumference $(\mathrm{cm})$ & 3.27 & $1.39-5.16$ & 0.001 \\
\hline BMI $\left(\mathrm{kg} / \mathrm{m}^{2}\right)$ & 1.54 & $0.52-2.55$ & 0.003 \\
\hline VAT area $\left(\mathrm{cm}^{2}\right)$ & 16.88 & $8.30-25.46$ & $<0.0001$ \\
\hline Body fat mass $(\mathrm{kg})$ & 2.49 & $0.65-4.32$ & 0.008 \\
\hline TNF- $\alpha(\mathrm{pg} / \mathrm{mL})$ & 0.98 & $0.13-1.82$ & 0.02 \\
\hline IL-8 $(\mathrm{pg} / \mathrm{mL})$ & 0.98 & $-1.09-3.01$ & 0.36 \\
\hline MCP-1 $(\mathrm{pg} / \mathrm{mL})$ & 25.73 & $-17.47-68.92$ & 0.24 \\
\hline
\end{tabular}

Estimates for the association between E-DII and body composition and inflammatory cytokines represent the regression coefficients for Path $\alpha$ Figure $2 \mathrm{~b}$. All estimates were adjusted for the potential effects of age. BMI, body mass index; DII, dietary inflammatory index; IL-8; Interleukin-8, MCP-1, monocyte chemotactic protein 1; TNF- $\alpha$, tumor necrosis factor alpha; VAT, visceral adipose tissue. 
Table 5. Regression coefficients (95\% CIs) for the associations between body composition and inflammatory cytokines and markers of T2D risk in black SA women.

\begin{tabular}{|c|c|c|c|c|c|c|c|c|c|}
\hline & \multicolumn{3}{|c|}{ Fasting Glucose } & \multicolumn{3}{|c|}{ Fasting Insulin } & \multicolumn{3}{|c|}{ HbA1c } \\
\hline & Estimate & $95 \% \mathrm{CI}$ & $p$-Value & Estimate & $95 \% \mathrm{CI}$ & $p$-Value & Estimate & $95 \% \mathrm{CI}$ & $p$-Value \\
\hline Waist circumference $(\mathrm{cm})$ & 0.01 & $-0.01-0.03$ & 0.22 & 0.40 & $0.23-0.57$ & $<0.0001$ & 0.01 & $0.00-0.02$ & 0.04 \\
\hline BMI $\left(\mathrm{kg} / \mathrm{m}^{2}\right)$ & 0.02 & $-0.02-0.06$ & 0.25 & 0.65 & $0.34-0.95$ & $<0.0001$ & 0.01 & $-0.00-0.03$ & 0.09 \\
\hline VAT area $\left(\mathrm{cm}^{2}\right)$ & 0.01 & $0.00-0.01$ & 0.02 & 0.10 & $0.06-0.13$ & $<0.0001$ & 0.00 & $0.00-0.00$ & 0.02 \\
\hline Body fat mass (kg) & 0.01 & $-0.01-0.03$ & 0.33 & 0.33 & $0.17-0.48$ & $<0.0001$ & 0.01 & $-0.00-0.01$ & 0.14 \\
\hline TNF- $\alpha$ (pg/mL) & 0.01 & $-0.01-0.03$ & 0.20 & 0.15 & $-0.04-0.34$ & 0.11 & -0.01 & $-0.02-0.00$ & 0.06 \\
\hline IL-8 (pg/mL) & -0.00 & $-0.01-0.01$ & 0.63 & -0.02 & $-0.13-0.09$ & 0.73 & -0.00 & $-0.01-0.00$ & 0.14 \\
\hline \multirow[t]{3}{*}{ MCP-1 (pg/mL) } & 0.00 & $-0.00-0.00$ & 0.29 & 0.00 & $-0.00-0.01$ & 0.56 & -0.00 & $-0.00-0.00$ & 0.92 \\
\hline & \multicolumn{3}{|c|}{ HOMA2-IR } & \multicolumn{3}{|c|}{ Two-Hour Glucose } & \multicolumn{3}{|c|}{ Matsuda Index * } \\
\hline & Estimate & $95 \% \mathrm{CI}$ & $p$-Value & Estimate & $95 \% \mathrm{CI}$ & $p$-Value & Estimate & $95 \% \mathrm{CI}$ & $p$-Value \\
\hline Waist circumference $(\mathrm{cm})$ & 0.06 & $0.03-0.09$ & $<0.0001$ & 0.04 & $0.01-0.08$ & 0.03 & 0.01 & $0.00-0.02$ & 0.008 \\
\hline BMI $\left(\mathrm{kg} / \mathrm{m}^{2}\right)$ & 0.10 & $0.04-0.15$ & $<0.0001$ & 0.06 & $-0.01-0.12$ & 0.10 & 0.02 & $0.00-0.03$ & 0.02 \\
\hline VAT area $\left(\mathrm{cm}^{2}\right)$ & 0.01 & $0.01-0.02$ & $<0.0001$ & 0.02 & $0.01-0.02$ & $<0.0001$ & 0.00 & $0.00-0.00$ & 0.001 \\
\hline Body fat mass $(\mathrm{kg})$ & 0.05 & $0.02-0.08$ & $<0.0001$ & 0.03 & $-0.01-0.06$ & 0.20 & 0.01 & $0.00-0.01$ & 0.03 \\
\hline TNF- $\alpha(\mathrm{pg} / \mathrm{mL})$ & 0.02 & $-0.00-0.05$ & 0.10 & 0.03 & $-0.02-0.07$ & 0.26 & 0.00 & $-0.00-0.01$ & 0.13 \\
\hline IL-8 (pg/mL) & -0.00 & $-0.02-0.01$ & 0.70 & -0.01 & $-0.03-0.01$ & 0.19 & -0.00 & $-0.00-0.00$ & 0.39 \\
\hline MCP-1 (pg/mL) & 0.00 & $-0.00-0.00$ & 0.49 & 0.00 & $-0.00-0.00$ & 0.16 & 0.00 & $-0.00-0.00$ & 0.58 \\
\hline
\end{tabular}

Regression coefficients correspond to Path $\beta$ in Figure $2 \mathrm{~b}$. All estimates were adjusted for the potential effects of age. BMI, body mass index; DII, dietary inflammatory index; HbA1c, glycated hemoglobin; HOMA2-IR, Homeostasis Model Assessment- insulin resistance; IL-8; Interleukin-8, MCP-1, monocyte chemotactic protein 1; TNF- $\alpha$, tumor necrosis factor alpha; VAT, visceral adipose tissue. ${ }^{*}$ Matsuda index was modelled as an inverse variable in order to calculate the proportion of mediation effect, thus the regression coefficients are positive instead of negative. 


\subsection{Associations between E-DII and Markers of T2D Risk in Black SA Women with Body Composition as Mediators}

The direct (Path $c^{\prime}$ ) and indirect effects (product of Paths $\alpha$ and $\beta, \alpha \beta$ ) of E-DII on markers of T2D risk, through body composition, including the proportion of mediation by the body composition measures also are presented in Table 2. A consistent finding was that VAT was a significant mediator of the association between E-DII and all markers of T2D risk, including fasting glucose and insulin, HbA1c, HOMA2-IR, two-hour glucose and Matsuda Index (all $p<0.05$ ). Both WC and BMI mediated the association between E-DII and fasting insulin, HOMA2-IR and Matsuda Index (all $p<0.05$ ). Body fat mass mediated the association between E-DII and fasting insulin and HOMA2-IR (all $p<0.05$ ). The proportion of the effect mediated by measures of central obesity (i.e., VAT and WC) was higher (23.5-100\%) than the proportion mediated by measures of total obesity, including BMI and body fat mass (10-60\%). Notably, VAT mediated most of the association between E-DII and markers of T2D risk, in particular for measures of insulin resistance (HOMA2-IR) and sensitivity (Matsuda Index) (proportion of mediation: 100\%). Furthermore, the proportion of the mediation effect for HOMA2-IR and Matsuda Index was higher (40-100\%) than for measures of glucose tolerance (e.g., fasting and two-hour glucose load). For instance, the mediating effects on the association between E-DII and HOMA2-IR were: For VAT (100.0\%), WC (80.0\%), BMI (60.0\%) and body fat mass (48.0\%) (Table 2).

Direct effects (Path c') between E-DII and T2D markers were not significant after adjusting for WC, BMI and VAT (Table 2). Similar findings were reported for body fat mass, except for the association between E-DII and two-hour glucose load.

\subsection{Direct and Indirect effects of E-DII on Markers of T2D in Black SA Women with Inflammatory Cytokines As Mediators}

The associations between E-DII and markers of T2D before and after adjustment for the effect of inflammatory cytokines on T2D risk are shown in Table 3. The inflammatory cytokines did not have any significant mediating effect on the association between E-DII and markers of T2D risk (Table 3). After adjusting for the inflammatory markers, E-DII was directly associated with fasting glucose (except for TNF- $\alpha$ ) and insulin, HbA1c, HOMA2-IR, two-hour glucose load and Matsuda Index (Table 3) $(p<0.05)$.

\section{Discussion}

This is the first study, to our knowledge, to suggest a link between diet-associated inflammation, as assessed by E-DII, and markers of T2D in a sample of middle-aged black SA women. Notably, we show that this association is mediated by adiposity, in particular central obesity (VAT and WC). Accordingly, measures of central adiposity were higher in participants with a positive E-DII score, reflecting a pro-inflammatory diet, compared to those with a negative E-DII score, which reflected an anti-inflammatory diet. While circulating TNF- $\alpha$ concentrations were higher in the positive E-DII group than the negative E-DII group, circulating inflammatory markers did not mediate the association between E-DII and markers of T2D in middle-aged black SA women.

Typical components of a Western diet, including high intakes of fat and red meat, and low intakes of vitamin A-rich fruit and vegetables, are linked to inflammation and a higher E-DII score [12,13] and have been associated with T2D and CVD risk in urban black SA populations [9-11]. These results suggest that pro-inflammatory foods are part of the habitual diet of urban black SA populations, and thus implicated in the pathophysiology of T2D in this population. Although the exact biological mechanisms implicated in the association between diet-induced inflammation and the development of T2D are not fully understood, we propose that excessive intake of pro-inflammatory foods may enhance fat accumulation, increase total and central obesity, in particular VAT, and subsequently induce an inflammatory profile, which is often reported in an obese state [16,17,37]. To substantiate this notion, the direct associations between E-DII and markers of T2D, after adjusting for measures of body composition, in particular VAT, were no longer significant. In contrast, the direct associations between E-DII and markers of T2D remained significant, after adjusting for the inflammatory cytokines. 
This suggests that a pro-inflammatory diet has a close association with measures of obesity, in particular for pro-inflammatory adipose tissues, such as VAT, and that the presence of obesity is more detrimental than that of low-grade inflammation in the development of T2D risk in this population.

Furthermore, the proposed mechanism for the development of an obese and/or inflammatory state in the presence of a pro-inflammatory diet is supported by the significantly higher measures of central adiposity and TNF- $\alpha$ levels in the positive E-DII score group compared to the negative E-DII group. Accordingly, obesity, in particular central obesity-related inflammation, has been implicated in the development of T2D [16,17,38]. In line with these findings, we reported that VAT mediated most of the association between E-DII and markers of T2D in comparison to other measures of adiposity. The higher proportion of mediation by measures of central obesity, in particular VAT, compared to measures of total adiposity (BMI as a proxy) might be explained by VAT having a higher inflammatory profile and higher rates of lipolysis than other adipose tissue sites [36,39,40]. Further, the anatomical position of VAT relative to the liver, leading to delivery of excess free fatty acids and pro-inflammatory cytokines (e.g., TNF- $\alpha$ ) via the hepatic portal system directly to the liver will impair hepatic insulin signaling $[36,39,40]$. Indeed, ectopic fat accumulation in the liver has been shown to impair many biological functions of the liver, and has been associated with hepatic IR, increased hepatic glucose production and reduced insulin-stimulated hepatic glucose uptake, thus increased risk for T2D [38-40].

Our finding that the association between diet-induced inflammation (assessed by the E-DII) and the risk of developing T2D is mediated by adiposity is notable, as only one study, to our knowledge, has reported both adiposity and low-grade inflammation as mediators in the association between diet-induced inflammation and markers of T2D in a European population [19]. However, the two studies are not easily comparable as two different versions of the DII were used; we used a new version of the DII with the updated scoring algorithm. Nonetheless, in our study, low-grade inflammation did not mediate the association between E-DII and markers of T2D. This can be explained by the non-significant association between inflammatory cytokines and markers of T2D risk, thus making a mediation effect "impossible". Moreover, we used three separate inflammatory cytokines, while a total/summary score of six inflammatory cytokines, including CRP, IL-6, 1L-8 and TNF- $\alpha$ was used as a marker of low-grade inflammation in the previous study [19].

Interestingly, out of the three inflammatory cytokines used in our analyses, only TNF- $\alpha$ was significantly different between the positive and negative E-DII score groups, and it also was associated with the E-DII score. TNF- $\alpha$ is the most highly secreted cytokine during obesity and was the first inflammatory cytokine linking obesity-induced inflammation to IR [41,42]. This suggests that TNF- $\alpha$ is a more prominent biomarker of obesity-induced inflammation than 1L-8 and MCP-1, in particular for this population, with high levels of obesity. However, the non-significant association between TNF- $\alpha$ and markers of T2D risk suggests that TNF- $\alpha$ (and other inflammatory cytokines) may not be directly associated with markers of T2D risk in this population or their effects might be diminished in the presence of more detrimental risk factors, such as obesity. Accordingly, it was reported that, despite a higher inflammatory profile at the genetic, adipose and circulating level, inflammation may not be the main driver of the higher T2D risk in black populations [43]. However, inflammation acts via paracrine and autocrine effects, limiting our ability to measure their specific effects. Nonetheless, results from our study and others suggest that other risk factors, such as body fat and its distribution, SES and lifestyle factors, including physical activity, as well as their interactions may be relatively more important risk factors for T2D in this population.

In our study the E-DII score ranged from -2.51 to +1.92 , which is narrower than that reported in a previous study of T2D ( -5.49 to +4.12$)$ [21]. The differences in the E-DII range between our study and the previous study may be explained by numerous factors, including differences in sample size, age, gender representation, type and number of food-parameters used in the calculation of the DII score and quantity of the food-parameters consumed. We also propose that the narrow range in the E-DII score might be explained by the homogeneity of the sample, in terms of SES and socio-cultural background (i.e., eating similar foods and calorically similar amounts), thus resulting in a low variety 
of the diet, especially when taking into account energy intake. Furthermore, it is also important to note that SES factors were not significantly different between the positive and negative E-DII score groups, implying that other external factors (e.g., socio-cultural factors) might be contributing to the pro- and anti-inflammatory profile of the diet. Furthermore, those individuals consuming a pro-inflammatory diet are at high risk of developing T2D, in particular those with central obesity.

This is the first study to investigate diet-associated inflammation in relation to T2D risk, in a high-risk and understudied African population, whose lifestyle differs from other populations. Importantly, we used DXA to provide a more accurate and objective measure of whole-body composition, including estimates of VAT. Despite its strengths, our study has potential limitations. This is a cross-sectional study, thus our results do not imply causality. Also, only three out of the eight possible inflammatory cytokines were detected in all the samples and used in the analysis, thus reducing the chance of finding significant results. Furthermore, the dietary intake was measured at a single time point, thus might not reflect the long-term or seasonal changes in dietary intake and lifestyle activities of the participants. In an attempt to control and reduce the confounding effects of total energy intake between the participants, the DII was adjusted per 1000 calories. Another potential weakness of the study is the use of only 21 food parameters out of 45 for the analysis of the DII score. However, the majority of earlier studies have not used all 45 food parameters [13,20,21]. For example, both Vahid et al. [20] and Denova-Gutierrez et al. [21] who have shown positive associations between DII and prediabetes or T2D risk used 27 food items out of the possible 45 food parameters. Nonetheless, positive associations between DII and inflammation have been reported even with fewer food parameters [13,20,21], supporting the robustness of the DII in investigating the association between diet-induced inflammation and inflammatory-related diseases. Furthermore, E-DII takes into account the entire diet of the individual and measures its inflammatory potential [14], making it a better tool compared to other dietary tools that only consider dietary patterns/food-groups and single-nutrients. Nonetheless, the DII score was calibrated against a global dietary database that did not include an African population [14]; which may not capture the true inflammatory profile of an African diet. Future refinements of the DII might include expansion of the global comparative database, which may help to capture even greater dietary diversity between populations.

\section{Conclusions}

In conclusion, our findings suggest that a pro-inflammatory diet may exacerbate the effects of obesity, in particular central obesity, and that a pro-inflammatory diet may increase the risk of developing T2D. Therefore, the promotion of healthy eating might prevent or reduce central obesity and obesity-induced inflammation, and subsequently lower the risk of developing T2D in black middle-aged black SA women.

Supplementary Materials: The following are available online at http:/www.mdpi.com/2072-6643/11/6/1246/s1, Table S1: Demographic, behavioral and lifestyle factors characteristics of the participants, Table S2: Nutrient intake between the positive and negative E-DII score groups.

Author Contributions: Designed or conceptualized the study, A.M., L.K.M., A.P.K., and J.H.G.; Conducted the research, performed biochemical and statistical analyses and wrote the first draft of manuscript, A.M.; Principal investigators and acquired funding for the study, L.K.M., E.C., T.O., and J.H.G.; Supervised the study and the writing of the manuscript, L.K.M., and J.H.G.; Performed the E-DII score analyses, N.S.; Are part of the researchers from the University of South Carolina that developed the DII, N.S. and J.R.H.; Critically reviewed and commented on the manuscript, L.K.M., E.C., T.O., N.S., J.R.H., A.P.K., and J.H.G. All authors read and approved the final version of the manuscript.

Funding: This research was funded by the Swedish Research Council (Swedish Development Grant, DNR: 2014-2522) the South African Medical Research Council, the Wellcome Trust of the United Kingdom (funded the Bt20+ cohort) and the National Research Foundation (NRF) of South Africa (scholarship for Asanda Mtintsilana, 111308).

Acknowledgments: The authors wish to thank the caregivers of Bt20 plus cohort for their participation in this study. We would also like to acknowledge the staff of the South African Medical Research Council (MRC)/University 
of the Witwatersrand (WITS) Developmental Pathways for Health Research Unit (DPHRU) at the Chris Hani Baragwanath Hospital in Soweto, Johannesburg, South Africa.

Conflicts of Interest: All authors declare no conflict of interest. Disclaimer: J.H. and N. Shivappa have the following disclosure: Professor James R. Hébert owns controlling interest in Connecting Health Innovations LLC (CHI), a company that has licensed the right to his invention of the dietary inflammatory index (DII) from the University of South Carolina in order to develop computer and smart phone applications for patient counselling and dietary intervention in clinical settings. N. Shivappa is an employee of CHI.

\section{References}

1. World Health Organisation. Global Status Report on Noncommunicable Diseases 2014; World Health Organization: Geneva, Switzerland, 2014; pp. 1-302.

2. Statistics South Africa. Mortality and Causes of Death in South Africa, 2016: Findings from Death Notification; Statistics South Africa: Pretoria, South Africa, 2018; Available online: https://doi.org/StatisticalreleaseP0309.3 (accessed on 27 March 2018).

3. International Diabetes Federation. Diabetes Atlas, 8th ed.; Cho, N.H., Kirigia, J., Claude, J., Ogurstova, M.K., Guariguata, L., Rathmann, W., Roglic, G., Forouhi, N., Dajani, R., Esteghamati, A., et al., Eds.; IDF: Brussels, Belgium, 2017.

4. Van der Merwe, M.T.; Crowther, N.J.; Schlaphoff, G.P.; Gray, I.P.; Joffe, B.I.; Lönnroth, P.N. Evidence for Insulin Resistance in Black Women from South Africa. Int. J. Obes. Relat. Metab. Disord. 2000, 24, 1340-1346. [CrossRef]

5. Goedecke, J.H.; Dave, J.A.; Faulenbach, M.V.; Utzschneider, K.M.; Lambert, E.V.; West, S.; Collins, M.; Olsson, T.; Walker, B.R.; Seckl, J.R.; et al. Insulin Response in Relation to Insulin Sensitivity- An Appropriate Beta-Cell Response in Black South African Women. Diabetes Care 2009, 32, 860-865. [CrossRef]

6. National Department of Health (NDoH); Statistics South Africa (Stats SA); South African Medical Research (SAMRC); ICF. South African Demographic and Health Survey (SADHS) 2016: Key Indicators; Statistics South Africa: Pretoria, South Africa, 2017.

7. Peer, N.; Steyn, K.; Lombard, C.; Lambert, E.V.; Vythilingum, B.; Levitt, N.S. Rising Diabetes Prevalence among Urban-Dwelling Black South Africans. PLoS ONE 2012, 7, e43336. [CrossRef]

8. Vorster, H.H.; Kruger, A.; Wentzel-Viljoen, E.; Kruger, H.S.; Margetts, B.M. Added Sugar Intake in South Africa: Findings from the Adult Prospective Urban and Rural Epidemiology Cohort Study. Am. J. Clin. Nutr. 2014, 99, 1479-1486. [CrossRef]

9. Peer, N.; Bradshaw, D.; Laubscher, R.; Steyn, N.; Steyn, K. Urban-Rural and Gender Differences in Tobacco and Alcohol Use, Diet and Physical Activity among Young Black South Africans between 1998 and 2003. Glob. Health Action 2013, 6, 1-10. [CrossRef]

10. Dolman, R.C.; Wentzel-Viljoen, E.; Jerling, J.C.; Feskens, E.J.; Kruger, A.; Pieters, M. The Use of Predefined Diet Quality Scores in the Context of CVD Risk during Urbanization in the South African Prospective Urban and Rural Epidemiological (PURE) Study. Public Health Nutr. 2013, 17, 1-11. [CrossRef]

11. Wentzel-Viljoen, E.; Lee, S.; Laubscher, R.; Vorster, H.H. Accelerated Nutrition Transition in the North West Province of South Africa: Results from the Prospective Urban and Rural Epidemiology (PURE-NWP-SA) Cohort Study, 2005 to 2010. Public Health Nutr. 2018, 2003, 1-12. [CrossRef]

12. Montonen, J.; Boeing, H.; Fritsche, A.; Schleicher, E.; Joost, H.G.; Schulze, M.B.; Steffen, A.; Pischon, T. Consumption of Red Meat and Whole-Grain Bread in Relation to Biomarkers of Obesity, Inflammation, Glucose Metabolism and Oxidative Stress. Eur. J. Nutr. 2013, 52, 337-345. [CrossRef]

13. Ruiz-Canela, M.; Zazpe, I.; Shivappa, N.; Hébert, J.R.; Sánchez-Tainta, A.; Corella, D.; Salas-Salvadó, J.; Fitó, M.; Lamuela-Raventós, R.M.; Rekondo, J.; et al. Dietary Inflammatory Index and Anthropometric Measures of Obesity in a Population Sample at High Cardiovascular Risk from the PREDIMED (PREvención Con DIeta MEDiterránea) Trial. Br. J. Nutr. 2015, 113, 984-995. [CrossRef]

14. Shivappa, N.; Steck, S.E.; Hurley, T.G.; Hussey, J.R.; Hébert, J.R. Designing and developing a literature-derived, population-based dietary inflammatory index. Public Health Nutr. 2014, 17, 1689-1696. [CrossRef]

15. Grosso, G.; Marventano, S.; Yang, J.; Micek, A.; Pajak, A.; Scalfi, L.; Galvano, F.; Kales, S.N. A Comprehensive Meta-Analysis on Evidence of Mediterranean Diet and Cardiovascular Disease: Are Individual Components Equal? Crit. Rev. Food Sci. Nutr. 2017, 57, 3218-3232. [CrossRef] [PubMed] 
16. Weisberg, S.P.; McCann, D.; Desai, M.; Rosenbaum, M.; Leibel, R.L.; Ferrante, A.W., Jr. Obesity Is Associated with Macrophage Accumulation in Adipose Tissue. Anaesth. Intensive Care 2003, 112, 1796-1808. [CrossRef]

17. Kim, C.S.; Park, H.S.; Kawada, T.; Kim, J.H.; Lim, D.; Hubbard, N.E.; Kwon, B.S.; Erickson, K.L.; Yu, R. Circulating Levels of MCP-1 and IL-8 Are Elevated in Human Obese Subjects and Associated with Obesity-Related Parameters. Int. J. Obes. 2006, 30, 1347-1355. [CrossRef]

18. Wirth, M.D.; Shivappa, N.; Davis, L.; Hurley, T.G.; Ortaglia, A.; Drayton, R.; Blair, S.N.; Hébert, J.R. Construct Validation of the Dietary Inflammatory Index among African Americans. J. Nutr. Heal. Aging 2017, 21, 487-491. [CrossRef] [PubMed]

19. Van Woudenbergh, G.J.; Theofylaktopoulou, D.; Kuijsten, A.; Ferreira, I.; Van Greevenbroek, M.M.; Van Der Kallen, C.J.; Schalkwijk, C.G.; Stehouwer, C.D.A.; Ocké, M.C.; Nijpels, G.; et al. Adapted Dietary Inflammatory Index and Its Association with a Summary Score for Low-Grade Inflammation and Markers of Glucose Metabolism: The Cohort Study on Diabetes and Atherosclerosis Maastricht (CODAM) and the Hoorn Study1-4. Am. J. Clin. Nutr. 2013, 98, 1533-1542. [CrossRef] [PubMed]

20. Vahid, F.; Shivappa, N.; Karamati, M.; Naeini, A.J.; Hebert, J.R.; Davoodi, S.H. Association between Dietary Inflammatory Index (DII) and Risk of Prediabetes: A Case-Control Study. Appl. Physiol. Nutr. Metab. 2017, 42, 399-404. [CrossRef]

21. Denova-Gutiérrez, E.; Muñoz-Aguirre, P.; Shivappa, N.; Hébert, J.R.; Tolentino-Mayo, L.; Batis, C.; Barquera, S. Dietary Inflammatory Index and Type 2 Diabetes Mellitus in Adults: The Diabetes Mellitus Survey of Mexico City. Nutrients 2018, 10, 385. [CrossRef]

22. Puoane, T.; Matwa, P.; Bradley, H.; Hughes, G. Socio-Cultural Factors Influencing Food Consumption Patterns in the Black African Population in an Urban Township in South Africa. Hum. Ecol. 2006, 14, 89-93.

23. Micklesfield, L.K.; Lambert, E.V.; Hume, D.J.; Chantler, S.; Pienaar, P.R.; Dickie, K.; Puoane, T.; Goedecke, J.H. Socio-Cultural, Environmental and Behavioural Determinants of Obesity in Black South African Women. Cardiovasc. J. Afr. 2013, 24, 369-375. [CrossRef]

24. Crowther, N.J.; Norris, S.A. The Current Waist Circumference Cut Point Used for the Diagnosis of Metabolic Syndrome in Sub-Saharan African Women Is Not Appropriate. PLoS ONE 2012, 7. [CrossRef]

25. Alberti, K.G.M.; Zimmet, P.; Shaw, J.; Grundy, S.M. The IDF Consensus Worldwide Definition of the Metabolic Syndrome. Diabet. Med. 2006, 23, 1-25.

26. World Health Organization. Obesity: Preventing and Managing the Global Epidemic; World Health Organization Technical Report Series; World Health Organization: Geneva, Switzerland, 2000; pp. 1-268.

27. Micklesfield, L.K.; Goedecke, J.H.; Punyanitya, M.; Wilson, K.E.; Kelly, T.L. Dual-Energy X-Ray Performs as Well as Clinical Computed Tomography for the Measurement of Visceral Fat. Obesity 2012, 20, 1109-1114. [CrossRef] [PubMed]

28. Micklesfield, L.K.; Reid, S.; Bewerunge, L.; Rush, E.C.; Goedecke, J.H. A Proposed Method to Measure Body Composition in Obese Individuals Using Dual-Energy X-Ray Absorptiometry. Int. J. Body Compos. Res. 2007, $5,147-151$.

29. Swindale, A.; Bilinsky, P. Development of a Universally Applicable Household Food Insecurity Measurement Tool: Process, Current Status, and Outstanding Issues. J. Nutr. 2006, 136, 1449S-1452S. [CrossRef] [PubMed]

30. Goedecke, J.H.; Mendham, A.E.; Clamp, L.; Nono Nankam, P.A.; Fortuin-De Smidt, M.C.; Phiri, L.; Micklesfield, L.K.; Keswell, D.; Woudberg, N.J.; Lecour, S.; et al. An Exercise Intervention to Unravel the Mechanisms Underlying Insulin Resistance in a Cohort of Black South African Women: Protocol for a Randomized Controlled Trial and Baseline Characteristics of Participants. J. Med. Internet Res. 2018, 20, 1-19. [CrossRef] [PubMed]

31. Nel, J.H.; Steyn, N. Report on South African Food Consumption Studies Undertaken among Population Groups (1983-2000): Average Intakes of Foods Most Commonly Consumed; Department of Health: Pretoria, South Africa, 2002.

32. Steyn, N.P.; Senekal, M.; Norris, S.A.; Whati, L.; Mackeown, J.M.; Nel, J.H. Europe PMC Funders Group Author Manuscript How Well Do Adolescents Determine Portion Sizes of Foods and Beverages? Asia Pac. J. Clin. Nutr. 2009, 15, 35-42.

33. Wallace, T.M.; Levy, J.C.; Matthews, D.R. Use and Abuse of HOMA Modeling. Diabetes Care 2004, 27, 1487-1495. Available online: https://www.dtu.ox.ac.uk/homacalculator/ (accessed on 5 September 2018). [CrossRef] [PubMed] 
34. Matsuda, M.; DeFronzo, R.A. Insulin Sensitivity Indices Obtained from Oral Glucose Tolerance Testing: Comparison with the Euglycemic Glucose Clamp. Diabetes Care 1999, 22, 1462-1470. Available online: http://mmatsuda.diabetes-smc.jp/english.html (accessed on 5 September 2018). [CrossRef] [PubMed]

35. Preacher, K.J.; Hayes, A.F. Asymptotic and Resampling Strategies for Assessing and Comparing Indirect Effects in Multiple Mediator Models. Behav. Res. Methods 2008, 40, 879-891. [CrossRef]

36. Ibrahim, M.M. Subcutaneous and Visceral Adipose Tissue: Structural and Functional Differences. Obes. Rev. 2010, 11, 11-18. [CrossRef]

37. Snel, M.; Jonker, J.T.; Schoones, J.; Lamb, H.; De Roos, A.; Pijl, H.; Smit, J.W.A.; Meinders, A.E.; Jazet, I.M. Ectopic Fat and Insulin Resistance: Pathophysiology and Effect of Diet and Lifestyle Interventions. Int. J. Endocrinol. 2012, 1-18. [CrossRef] [PubMed]

38. Pou, K.M.; Massaro, J.M.; Hoffmann, U.; Vasan, R.S.; Maurovich-Horvat, P.; Larson, M.G.; Keaney, J.F.; Meigs, J.B.; Lipinska, I.; Kathiresan, S.; et al. Visceral and Subcutaneous Adipose Tissue Volumes Are Cross-Sectionally Related to Markers of Inflammation and Oxidative Stress: The Framingham Heart Study. Circulation 2007, 116, 1234-1241. [CrossRef] [PubMed]

39. Perry, R.J.; Camporez, J.P.G.; Kursawe, R.; Titchenell, P.M.; Zhang, D.; Perry, C.J.; Jurczak, M.J.; Abudukadier, A.; Han, M.S.; Zhang, X.M.; et al. Hepatic Acetyl CoA Links Adipose Tissue Inflammation to Hepatic Insulin Resistance and Type 2 Diabetes. Cell 2015, 160, 745-758. [CrossRef] [PubMed]

40. Bonora, E. Relationship between Regional Fat Distribution and Insulin Resistance. Int. J. Obes. 2000, 24 (Suppl. 2), S32-S35. [CrossRef]

41. Gregor, M.F.; Hotamisligil, G.S. Inflammatory Mechanisms in Obesity. Annu. Rev. Immunol. 2011, $29,415-445$. [CrossRef]

42. Hotamisligil, G.S.; Shargill, N.S.; Spiegelman, B.M. Adipose Expression of Tumor Necrosis Factor-x: Direct Role in Obesity-Linked Insulin Resistance. Science 1993, 259, 87-92. [CrossRef]

43. Evans, J.; Goedecke, J.H.; Söderström, I.; Burén, J.; Alvehus, M.; Blomquist, C.; Jonsson, F.; Hayes, P.M.; Adams, K.; Dave, J.A.; et al. Depot- and Ethnic-Specific Differences in the Relationship between Adipose Tissue Inflammation and Insulin Sensitivity. Clin. Endocrinol. (Oxf.) 2011, 74, 51-59. [CrossRef] 